\title{
Lithocholic Acid Activates Mas-Related G Protein-Coupled Receptors, Contributing to Itch in Mice
}

\author{
Myung-Hyun Song ${ }^{1}$ and Won-Sik Shim ${ }^{1,2, *}$ \\ ${ }^{1}$ College of Pharmacy, Gachon University, Incheon 21936, \\ ${ }^{2}$ Gachon Institute of Pharmaceutical Sciences, Incheon 21936, Republic of Korea
}

\begin{abstract}
The present study focused on lithocholic acid (LCA), a secondary bile acid that contributes to cholestatic pruritus. Although recent studies have found that LCA acts on MAS-related G protein-coupled receptor family member X4 (MRGPRX4) in humans, it is unclear which subtypes of MRGPRs are activated by LCA in mice since there is no precise ortholog of human MRGPRX4 in the mouse genome. Using calcium imaging, we found that LCA could activate mouse Mrgpra1 when transiently expressed in HEK293T cells. Moreover, LCA similarly activates mouse Mrgprb2. Importantly, LCA-induced responses showed dose-dependent

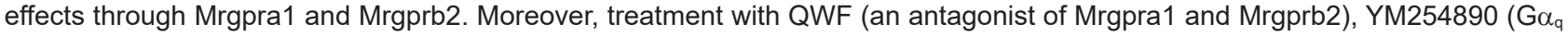
inhibitor), and U73122 (an inhibitor of phospholipase C) significantly suppressed the LCA-induced responses, implying that the LCA-induced responses are indeed mediated by Mrgpra1 and Mrgprb2. Furthermore, LCA activated primary cultures of mouse sensory neurons and peritoneal mast cells, suggesting that Mrgpra1 and Mrgprb2 contribute to LCA-induced pruritus. However, acute injection of LCA did not induce noticeable differences in scratching behavior, implying that the pruritogenic role of LCA may be marginal in non-cholestatic conditions. In summary, the present study identified for the first time that LCA can activate Mrgpra1 and Mrgprb2. The current findings provide further insight into the similarities and differences between human and mouse MRGPR families, paving a way to understand the complex roles of these pruriceptors.
\end{abstract}

Key Words: Lithocholic acid, Bile acid, Pruritus, MRGPRX4, Mrgpra1, Mrgprb2

\section{INTRODUCTION}

Lithocholic acid (LCA) or 3 $\alpha$-hydroxy-5 $\beta$-cholan-24-oic acid is a hydrophobic secondary bile acid that is mostly produced by the $7 \alpha$-dehydroxylation of chenodeoxycholic acid (CDCA), a primary bile acid. (Ridlon et al., 2016). LCA is often regarded as a toxic compound since the administration of LCA induces intrahepatic cholestasis (Fisher et al., 1971; Woolbright et al., 2014). Moreover, LCA levels are significantly elevated in patients with chronic cholestatic liver disease (Carey et al., 1966). Likewise, administration of LCA into mice also induced liver injury, which is often found in cholestatic liver diseases (Fickert et al., 2006).

Cholestasis is defined as an impairment of bile flow that accompanies various indications related to the liver, such as detoxification, excretion, and/or digestion. The major symptoms of cholestasis include fatigue, jaundice, dark urine, lightcolored stool, steatorrhea, and pruritus. Pruritus may be an unexpected symptom since it is not known how cholestasis can induce pruritus for years. Fortunately, recent advances have elucidated the underlying mechanisms of cholestatic pruritus, emphasizing the role of bile acids in the pathogenesis of cholestatic pruritus (Sanjel and Shim, 2020). Importantly, it was recently revealed that MRGPRX4 is a bile acid receptor responsible for human cholestatic itch (Meixiong et al., 2019c; Yu et al., 2019). Deoxycholic acid (DCA) is the most potent bile acid that can activate MRGPRX4 (Meixiong et al., 2019c; Yu et al., 2019). Remarkably, LCA can also marginally activate MRGPRX4, leading to a weak, albeit evident, itch sensation in humans (Yu et al., 2019). Therefore, LCA is also a bile acid responsible for cholestatic pruritus.

Mas-related G protein-coupled receptor (MRGPR) is a family of G-protein coupled receptors (GPCRs) that encodes a receptor required for mostly chronic, histamine-independent pruritus. Strangely enough, MRGPRs are not well conserved across different species, especially between mice and hu-

\section{Open Access https://doi.org/10.4062/biomolther.2021.059}

This is an Open Access article distributed under the terms of the Creative Commons Attribution Non-Commercial License (http://creativecommons.org/licenses/by-nc/4.0/) which permits unrestricted non-commercial use, distribution, and reproduction in any medium, provided the original work is properly cited.
Received Mar 29, 2021 Revised May 7, 2021 Accepted Jun 1, 2021

Published Online Jul 15, 2021

\section{*Corresponding Author}

E-mail: wsshim@gachon.ac.kr

Tel: +82-32-820-4960, Fax: +82-32-820-4829 
mans. Studies have found that mice have numerous distinct subfamilies of MRGPR known as Mrgpra (a1-a12, a14, a16, a19), Mrgprb (b1-b6, b8, b10, b11), and Mrgprc (c11) (Dong et al., 2001), whereas humans only have four corresponding MRGPR genes (MRGPRX1-4). This indicates that there is no simple one-to-one ortholog of human MRGPRX in mice. Rather, mice are likely to have many functionally similar, and even overlapping "counterpart" genes that play comparable roles compared to MRGPRX. Indeed, mouse Mrgprb2 is functionally analogous to human MRGPRX2 in mast cells (Subramanian et al., 2016). Mouse Mrgprc11 is often regarded as a counterpart of human MRGPRX1 for similar reasons (Mrgprc11 is also called mouse Mrgprx1). For human MRGPRX4, mouse Mrgpra1 may be a functional ortholog since both human MRGPRX4 and mouse Mrgpra1 are activated by bilirubin (Meixiong et al., 2019b; Lay and Dong, 2020). Sequence similarity indicates that mouse Mrgprb2 shares high similarities with human MRGPRX4 (Supplementary Fig. 1), suggesting a possible overlap in functions.

Although it was previously reported that LCA activates human MRGPRX4 (Yu et al., 2019), it has not been investigated whether LCA can activate MRGPRs in mice. Therefore, in the present study, we have specifically focused on determining whether LCA can activate mouse Mrgpra1 and Mrgprb2, which are similar to MRGPRX4. As activation of both Mrgpra1 and Mrgrpb2 can lead to transient elevation of intracellular calcium levels (Azimi et al., 2016), responses to LCA were measured using the calcium imaging technique. Furthermore, we checked whether LCA can activate itch-related sensory neurons and mast cells in an MRGPR-dependent manner in mice.

\section{MATERIALS AND METHODS}

\section{Reagents}

Lithocholic acid (LCA), deoxycholic acid (DCA), taurolithocholic acid (TLCA), compound 48/80, IL-3, U-73122, SKF-96365, and 4-Nitrophenyl N-acetyl- $\beta$-D-glucosaminide (pNAG) were purchased from Sigma-Aldrich (St. Louis, MO, USA). YM-254890 was purchased from FUJIFILM Wako (Osaka, Japan). QWF (Boc-GIn-D-Trp(Formyl)-Phe benzyl ester trifluoroacetate) was obtained from Tocris Bioscience (Bristol, UK). Murine stem cell factor (SCF) was purchased from Peprotech (Cranbury, NJ, USA).

\section{Gene cloning}

Total RNA from mouse dorsal root ganglia (DRG) and skin was isolated using the Easy-spin ${ }^{\mathrm{TM}}$ Total RNA Extraction Kit (iNtRON Biotechnology Inc., Seongnam, Korea). First-strand cDNA was then produced from total RNA from both DRG and skin using the PrimeScript ${ }^{\mathrm{TM}}$ 1st strand cDNA Synthesis Kit (Takara, Shiga, Japan).

The full-length coding sequence of mouse Mrgpra1 was cloned by PCR from mouse DRG cDNA using the forward (5'CAG CAC AGT GGC GGC CAC CAT GGG GGA AAG CAG CAC C-3') and reverse (5'-GGG CCC TCT AGA CTC GAG CGG CCT CAT GGC TCT GAT TTG CTT CT-3') primers. Similarly, the full-length coding sequence of mouse Mrgprb2 was cloned from mouse skin cDNA using the forward (5'-ATA TCC AGC ACA GTG GCG GCC ACC ATG AGT GGA GAT TTC CTA ATC AAG-3') and reverse (5'-GCC CTC TAG ACT CGA GCG GCC TCA GCT GCA GCT CTG AAC AGT TT-3') primers. The
PCR products of Mrgpra1 and Mrgprb2 were subcloned into pcDNA3.1 using the modified protocol of single step ligation independent cloning (SLIC) (Jeong et al., 2012; Islam et al., 2017). Sequencing of both Mrgpra1 and Mrgprb2 cDNA resulted in $100 \%$ identity with sequences registered in NCBI GenBank (NM_153095 and NM_175531, respectively). Human MRGPRX2 (plasmid \#66440) and MRGPRX4 (plasmid \#66442) cDNAs were purchased from Addgene (Watertown, MA, USA) and subcloned into pcDNA3.1.

\section{Cell culture and gene transfection}

HEK293T cells were cultured in Dulbecco's Modified Eagle Medium (DMEM, Gibco, Life Technologies, Grand Island, NY, USA) containing $10 \%$ fetal bovine serum (FBS, Gibco) and $1 \%$ ZellShield $^{\circledR}$ (Minerva Biolabs, Berlin, Germany). Cells were transfected with genes using FuGENE ${ }^{\circledR}$ HD Transfection Reagent (Promega, Madison, WI, USA) according to the manufacturer's instructions. Twenty-four h after gene transfection, calcium imaging experiments were performed.

\section{Primary culture of DRG neurons}

DRG neurons were isolated and cultured as previously described (Pradhananga and Shim, 2015). In detail, DRG neurons were incubated for $40 \mathrm{~min}$ at $37^{\circ} \mathrm{C}$ with $1.2 \mathrm{mg} / \mathrm{mL}$ collagenase (Worthington Biochemical, Lakehold, NJ, USA), followed by an additional 40 min incubation at $37^{\circ} \mathrm{C}$ with 2.5 $\mathrm{mg} / \mathrm{mL}$ trypsin (Gibco). After incubation, cells were centrifuged at $30 \mathrm{~g}$ for $10 \mathrm{~min}$ and resuspended in neurobasal medium (Gibco, Life Technologies) containing 10\% FBS, 50-100 ng/ $\mathrm{mL}$ nerve growth factor (Invitrogen, Gaithersburg, MD, USA), and $100 \mathrm{U} / \mathrm{mL}$ ZellShield ${ }^{\circledR}$. Cells were then plated on poly Llysine-treated 8-well Lab-Tek chambers (Thermo Fisher Scientific, Waltham, MA, USA) and incubated for $48 \mathrm{~h}$ in the presence of $95 \%$ humidity and $5 \% \mathrm{CO}_{2}$ at $37^{\circ} \mathrm{C}$.

\section{Isolation and culture of peritoneal mast cell (PMCs)}

PMCs were isolated by washing the peritoneal cavity using the peritoneal lavage technique (Tsvilovskyy et al., 2018). Briefly, cells were centrifuged at $300 \mathrm{~g}$ and resuspended in RPMI 1640 medium (Gibco, Life Technologies) supplemented with $20 \%$ FBS, $1 \%$ penicillin-streptomycin (Pen-Strep, Gibco), $10 \mathrm{ng} / \mathrm{mL}$ murine IL-3 (Sigma-Aldrich, St. Louis, MO, USA), and $30 \mathrm{ng} / \mathrm{mL}$ murine stem cell factor (SCF; Peprotech). The cells were further cultured in $5 \% \mathrm{CO}_{2}$ at $37^{\circ} \mathrm{C}$. On day 2 of cultivation, all non-adherent cells were discarded, and the medium was changed. The cells were split and plated in T25 flasks and poly L-lysine-treated 8-well Lab-Tek chambers (Thermo Fisher Scientific) on day 9. PMCs were used for calcium imaging and $\beta$-hexosaminidase release assay on days 11 and 13 , respectively.

\section{Calcium imaging: measurement of intracellular $\mathrm{Ca}^{2+}$ changes}

Intracellular $\mathrm{Ca}^{2+}$ changes were measured using a fluorescence microscope (DMi8, Leica, Wetzlar, Germany). Cells were loaded with $5 \mu \mathrm{M}$ Fluo-3/AM (Invitrogen, Eugene, OR, USA) and incubated for $40 \mathrm{~min}$ at $37^{\circ} \mathrm{C}$. Then, cells were washed with normal bath solution (140 mM NaCl, $5 \mathrm{mM} \mathrm{KCl}$, $2 \mathrm{mM} \mathrm{CaCl} 2,0.5 \mathrm{mM} \mathrm{MgCl}_{2}, 10 \mathrm{mM}$ glucose, and $5.5 \mathrm{mM}$ HEPES, adjusted to $\mathrm{pH}$ 7.4). After incubation, the medium was washed out, and compounds were added to increase intracellular calcium levels. Fluorescence was detected at 
excitation and emission wavelengths of 488 and $515 \mathrm{~nm}$, respectively. Fluorescent microscopic images were recorded on a computer for $3 \mathrm{~min}$ at time intervals of $3.0 \mathrm{~s}$. Image analysis was performed using ImageJ ( $\mathrm{NIH}$, Bethesda, MD, USA) with custom-made scripts for automatic cell count and image production. Intracellular $\mathrm{Ca}^{2+}$ changes were expressed as $\mathrm{F} /$ $F_{0}$, where $F$ indicates the intensity of fluorescence, and $F_{0}$ indicates the initial fluorescence intensity.

\section{$\boldsymbol{\beta}$-hexosaminidase release assay with mouse PMCs}

Mouse PMCs were centrifuged at $300 \mathrm{~g}$ for $5 \mathrm{~min}$ and resuspended in a normal bath solution $(140 \mathrm{mM} \mathrm{NaCl}, 5 \mathrm{mM}$ $\mathrm{KCl}, 2 \mathrm{mM} \mathrm{CaCl}_{2}, 0.5 \mathrm{mM} \mathrm{MgCl}_{2}, 10 \mathrm{mM}$ glucose, and $5.5 \mathrm{mM}$ HEPES, adjusted to $\mathrm{pH}$ 7.4). Cells were seeded in a V-bottom 96-well plate. All experiments were performed in duplicate. Degranulation was induced by incubation of PMCs in the presence of the agonists for $1 \mathrm{~h}$ at $37^{\circ} \mathrm{C}$ and $5 \% \mathrm{CO}_{2}$. Cells were then centrifuged at $200 \mathrm{~g}$ for $5 \mathrm{~min}$ at $4^{\circ} \mathrm{C}$. The supernatants were separated, and the cell pellets were lysed in a normal bath solution supplemented with $1 \%$ Triton-X 100 for 5 min at room temperature. The amount of released $\beta$-hexosaminidase enzyme was quantified by spectrophotometric analysis of pNAG hydrolysis, as previously described (Tsvilovskyy et al., 2018). Briefly, the cell lysates and supernatants were incubated separately with $2 \mathrm{mM}$ pNAG for $1 \mathrm{~h}$ at $37^{\circ} \mathrm{C}$, and the reaction was stopped by adding $200 \mathrm{mM}$ glycine $(\mathrm{pH} \mathrm{10.7,} \mathrm{with}$ $\mathrm{NaOH}$ ). The hydrolysis rate of pNAG was quantified by colorimetric measurements at $405 \mathrm{~nm}$ using the Synergy $\mathrm{H} 1$ Hybrid
Multi-Mode Reader (BioTek, Winooski, VT, USA). For background correction, the absorbance at $630 \mathrm{~nm}$ was subtracted from that at $405 \mathrm{~nm}$. The percentage of $\beta$-hexosaminidase release was calculated as the absorbance ratio of the supernatant to the sum of the supernatant and lysate.

\section{In vivo mouse scratching behavior experiments}

All animal experimental procedures were performed in accordance with the animal protocol approved by the Institutional Animal Care and Use Committee of Gachon University, Incheon, Korea (Approved animal protocol number: GIACUCR2020002). Ten-week-old male ICR mice were purchased from Orient Laboratory Animals (Seoul, Korea). The animals were allowed free access to food and water under a 12:12 $\mathrm{h}$ light:dark cycle. To elicit scratching behavior, $10 \mu \mathrm{L}$ of LCA $(50 \mu \mathrm{g} / \mathrm{site})$ was administered via an intradermal (i.d.) injection into the right cheek. To verify the itch mechanism of LCA, 10 $\mu \mathrm{L}$ of LCA and $500 \mu \mathrm{M}$ QWF mixture were co-administered intradermally to the cheek injection model. To minimize irritation, mice were not shaved before injections. All experiments were video-recorded, and bouts of scratching using the hind limbs were counted immediately after the i.d. injection for up to $30 \mathrm{~min}$.

\section{Statistical analysis}

All data are presented as the mean \pm standard error of the mean (SEM). The Student's t-test was used for comparison between two groups. One-way analysis of variance (ANOVA)
A
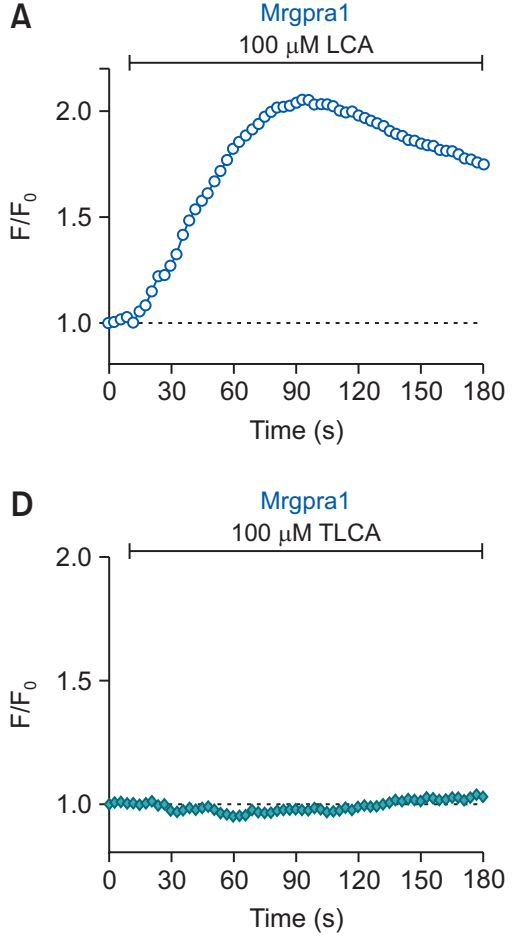

B

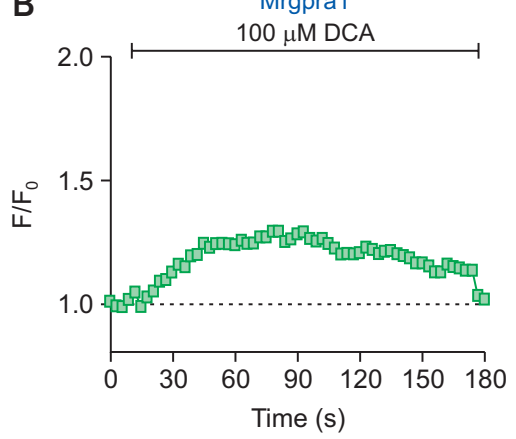

E

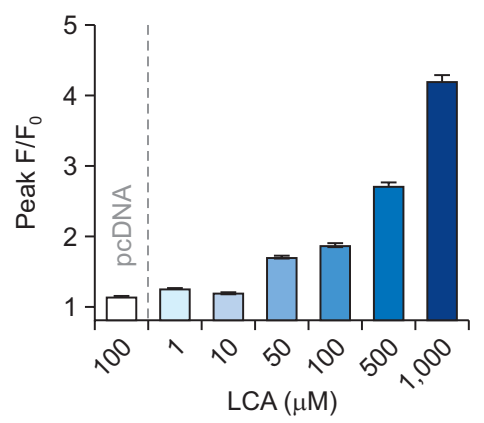

C

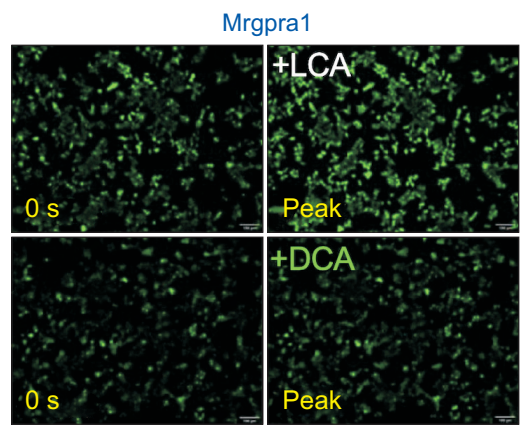

$\mathbf{F}$

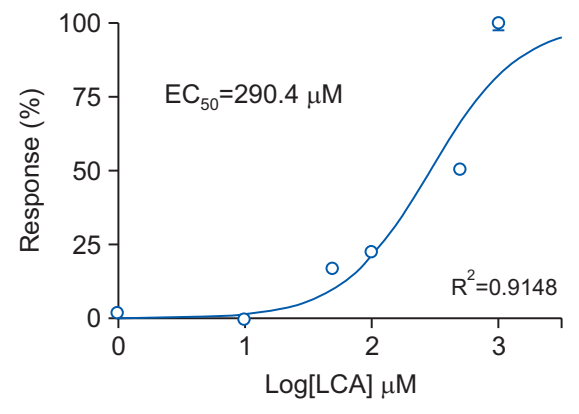

Fig. 1. Lithocholic acid (LCA) induces an increase in intracellular calcium levels via Mrgpra1. (A, B) HEK293T cells transiently expressing mouse Mrgpra1 (Mrgpra1-HEK293T) were treated with either LCA $(100 \mu \mathrm{M})$ or deoxycholic acid (DCA; $100 \mu \mathrm{M})(\mathrm{n}=2,932 \mathrm{cells}$ and $\mathrm{n}=2,312$ cells, respectively). (C) Representative fluorescent images of Mrgpra1-HEK293T at initial (0) and peak time points. Scale bar=100 $\mu \mathrm{m}$. (D) Taurolithocholic acid (TLCA; $100 \mu \mathrm{M}$ ) did not induce any noticeable responses in Mrgpra1-HEK293T ( $n=3,216$ cells). (E) A summary of peak $\mathrm{F} / \mathrm{F}_{0}$ from Mrgpra1-HEK293T after treatment with various concentrations of LCA. Cells without Mrgpra1 expression did not show an increase in intracellular calcium levels ( $p c D N A, n=1,684$ cells). ( $F$ ) Concentration-effect curves with LCA on Mrgpra1-HEK293T $\left(R^{2}=0.9148\right)$. 
with Dennett's multiple comparison post-test was used for comparison among more than three groups. Each concentration-response curve was fitted, and each $\mathrm{EC}_{50}$ was calculated using GraphPad Prism ${ }^{\circledR}$ software (GraphPad, San Diego, CA, USA).

\section{RESULTS}

\section{LCA increases intracellular calcium levels via Mrgpra1}

Before investigating the effect of mouse Mrgpra1, we first examined whether Mrgpra1 is functionally active when transiently expressed in HEK293T cells. For this reason, HEK293T cells expressing Mrgpra1 (Mrgpra1-HEK293T) were treated with compound $48 / 80$, a compound that can activate both mouse Mrgpra1 and Mrgprb2 (Azimi et al., 2016). As expected, treatment with compound 48/80 $(100 \mu \mathrm{M})$ significantly increased the intracellular fluorescence intensity (Supplementary Fig. 2A). Moreover, treatment with compound $48 / 80$ did not induce any changes in the intracellular fluorescence intensity in cells expressing the vector plasmid pcDNA3.1 alone (data not shown). Thus, Mrgpra1-HEK293T was functionally active, supporting the validity of the following experiments.

We also verified whether LCA and DCA were chemically active. Since human MRGPRX4 can be activated by LCA and DCA (Yu et al., 2019), MRGPRX4 as a positive control was transiently expressed in HEK293T cells, and both LCA and DCA were applied. As a result, we also observed the exact same responses, as DCA and LCA both activated MRGPRX4 (Supplementary Fig. 3A, 3B), demonstrating that LCA and DCA were effective. We also expressed human MRGPRX2 in HEK293T cells and found that LCA and DCA were not potent agonists of MRGRPX2 (Supplementary Fig. 3C, 3D).

When $100 \mu \mathrm{M}$ LCA was added to Mrgpra1-HEK293T, as shown in Fig. $1 \mathrm{~A}$ and $1 \mathrm{C}$, a significant increase in intracellular fluorescence intensity levels was observed, implying that LCA activates Mrgpra1. Treatment with DCA (Supplementary Fig. 2D), a structurally similar bile acid, also elevated the fluorescence intensity in Mrgpra1-HEK293T, but to a lesser extent (Fig. 1B, 1C). Intriguingly, $100 \mu \mathrm{M}$ taurolithocholic acid (TLCA) failed to activate Mrgpra1 (Fig. 1D), indicating the specificity of response to bile acids by Mrgpra1.

To further verify whether the LCA-induced responses were indeed mediated by Mrgpra1, dose-response experiments were performed. Results showed that LCA induced dose-dependent responses in Mrgpra1-HEK293T (Fig. 1E), with EC $\mathrm{C}_{50}$ value as $290.4 \mu \mathrm{M}$ (Fig. 1F). Again, cells transfected with the pcDNA3.1 alone did not show any changes when incubated with $100 \mu \mathrm{M}$ LCA (pcDNA in Fig. 1E). Taken together, LCA can evoke intracellular calcium increase in Mrgpra1-HEK293T, suggesting that LCA can activate Mrgpra1.

\section{Mrgpra1 specifically mediates the LCA-induced response}

To clarify whether the response is due to Mrgpra1 activation, $1 \mu \mathrm{M}$ QWF, a peptide that can block Mrgpra1 activation (Azimi et al., 2016), was pretreated 5 min before LCA appli-
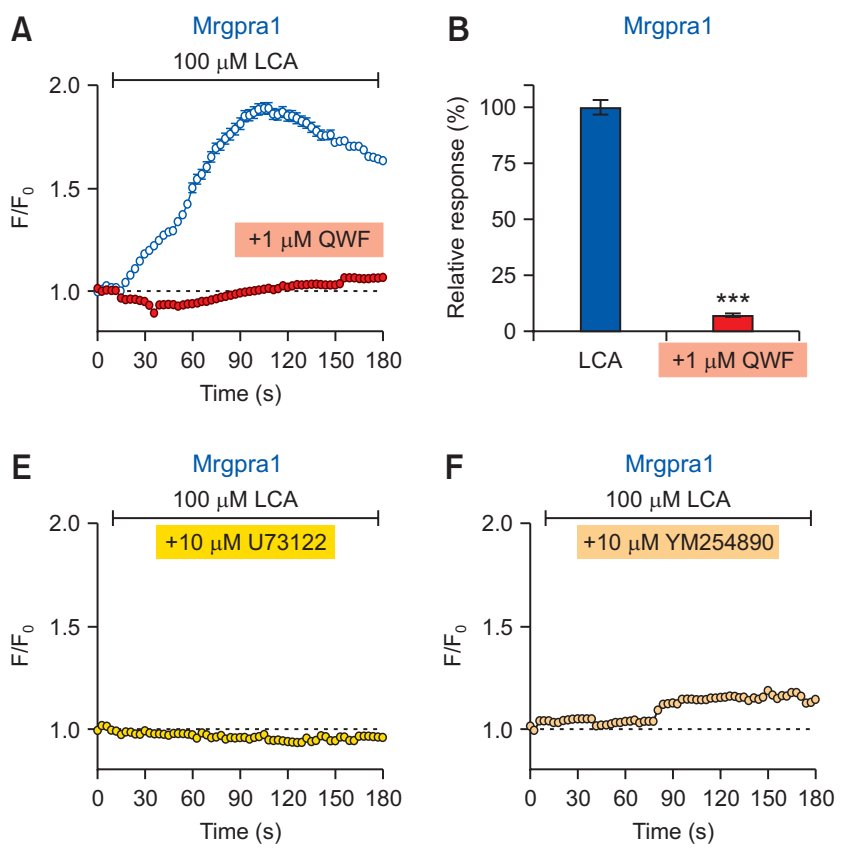
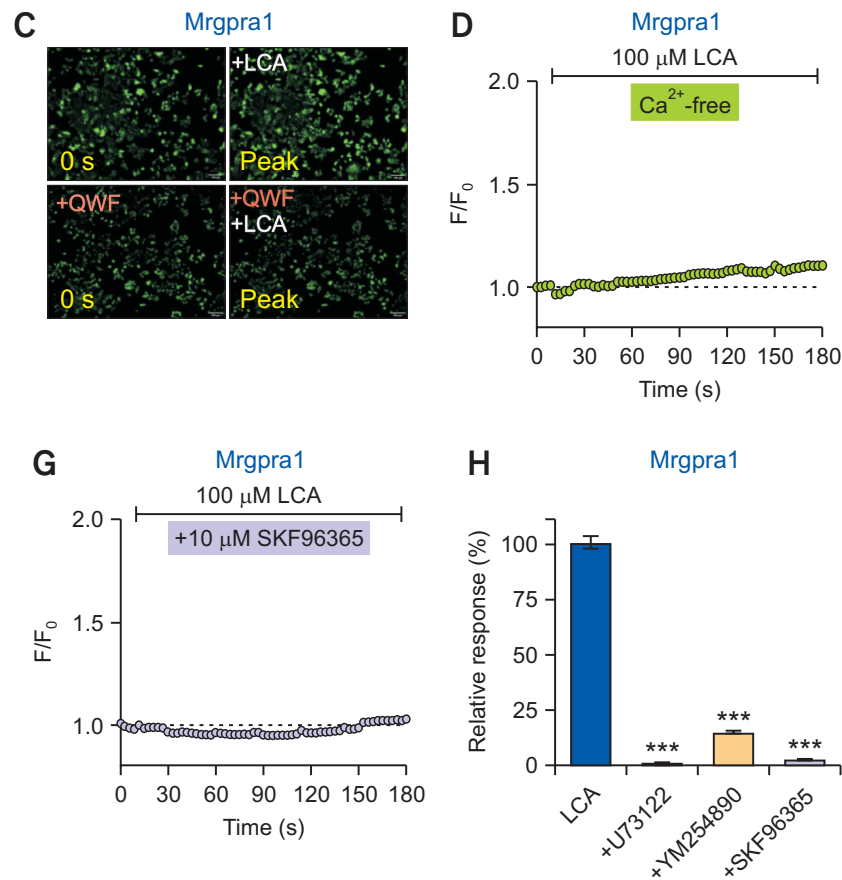

Fig. 2. Lithocholic acid (LCA)-induced response is specifically mediated by Mrgpra1. (A) The LCA-induced intracellular calcium increase ( $n=1,342$ cells) was suppressed by pretreatment with $1 \mu M$ QWF ( $n=1,767$ cells) in Mrgpra1-expressing HEK293T cells (Mrgpra1HEK293T). (B) A comparison of relative responses between LCA vs. QWF pretreatment. ${ }^{* * *} p<0.001$. (C) Representative fluorescent images of Mrgpra1-HEK293T at initial (0 s) and peak time points for QWF pretreatment before LCA application. Scale bars $=100 \mu \mathrm{m}$. (D) LCA failed to evoke an increase in intracellular calcium levels in calcium-free media ( $n=3,900$ cells). (E-G) Responses from various inhibitors on LCA treatment in Mrgpra1-HEK293T. LCA-induced responses were significantly suppressed by SKF96365 (E; a non-specific TRPC blocker, 1028 cells), U73122 (F; an inhibitor of PLC, $n=969$ cells), and YM254890 (G; a G $\alpha_{q}$ inhibitor, $n=914$ cells). (H) A summary of relative responses of pretreatment effects with various inhibitors in the Mrgpra1-HEK293T. ${ }^{* * *} p<0.001$. 
cation. As shown in Fig. 2A, 2B, and 2C, QWF pretreatment almost completely abolished the increase in the LCA-induced calcium levels, indicating that the observed calcium increase by LCA is likely mediated by Mrgpra 1 .

To further verify the source of intracellular calcium increase, external media lacking calcium $\left(\mathrm{Ca}^{2+}\right.$-free) was prepared. As shown in Fig. 2D, the calcium-free condition did not evoke any noticeable intracellular calcium increase by LCA in Mrgpra1HEK293T. This result suggests that the LCA-induced intracellular calcium increase is due to an influx from extracellular media, implying the involvement of certain calcium-permeable ion channels endogenously expressed in HEK293T cells.

Mrgpra1 is activated via the $\mathrm{G} \alpha_{\mathrm{q} / 11}$ signaling pathway (Han et al., 2002). Hence, activation of Mrgpra1 leads to phospholipase $C$ (PLC) stimulation. To confirm this, pretreatment with U73122, an inhibitor of PLC, was applied before LCA treatment in Mrgpra1-HEK293T. As expected, pretreatment with U73122 significantly blocked the LCA-induced calcium influx in Mrgpra1-HEK293T (Fig. 2E, 2H). Moreover, pretreatment with YM254890, a $G \alpha_{q}$ inhibitor, also significantly abolished the LCA-induced calcium influx in Mrgpra1-HEK293T (Fig. 2F, $2 \mathrm{H})$. Furthermore, pretreatment with SKF96365, an inhibitor of store-operated $\mathrm{Ca}^{2+}$ entry such as calcium-permeable TRPC ion channels, also blocked the LCA-induced calcium influx in Mrgpra1-HEK293T (Fig. 2G, 2H), implying that Mrgpra1 may take advantage of TRPC for calcium influx, at least in the current experimental setup. Overall, it was confirmed that LCA could stimulate Mrgpra1, which leads to calcium influx in a
$\mathrm{G}_{\mathrm{q} / 11}$ - and PLC-dependent manner.

\section{LCA induces intracellular calcium increase via Mrgprb2}

A similar approach was applied to mouse Mrgprb2. As shown in Supplementary Fig. 2B, Mrgprb2 was also found to be functionally active when transiently expressed in HEK293T cells (Mrgprb2-HEK293T), as verified by treatment with compound 48/80, which is also an agonist for Mrgprb2 (McNeil et al., 2015; Azimi et al., 2016).

Surprisingly, $100 \mu \mathrm{M}$ LCA also evoked a strong intracellular calcium level increase in Mrgprb2-HEK293T, which was not expected initially (Fig. 3A, 3C). Similar to Mrgpra1, DCA treatment also induced a marginal increase (Fig. 3B, 3C), whereas TLCA barely showed noticeable responses (Fig. 3D).

Similar to Mrgpra1-HEK293T, the intracellular calcium rise by LCA in Mrgprb2-HEK293T also showed a dose-dependent manner (Fig. 3E) with an $\mathrm{EC}_{50}$ value of $183.3 \mu \mathrm{M}$ (Fig. 3F). In contrast, cells transfected with pcDNA3.1 alone did not show any intracellular calcium changes to $100 \mu \mathrm{M}$ LCA (pcDNA in Fig. 3E). Taken together, these results indicate that LCA can also activate Mrgprb2.

\section{Mrgprb2 specifically mediates the LCA-induced response}

To further verify whether the LCA-induced response is due to Mrgprb2 activation, QWF was used because it suppresses Mrgprb2 activation (Azimi et al., 2016). Pretreatment with 10 $\mu \mathrm{M}$ QWF significantly inhibited the LCA-induced responses in Mrgprb2-HEK293T (Fig. 4A-4C), indicating that the LCA-
A

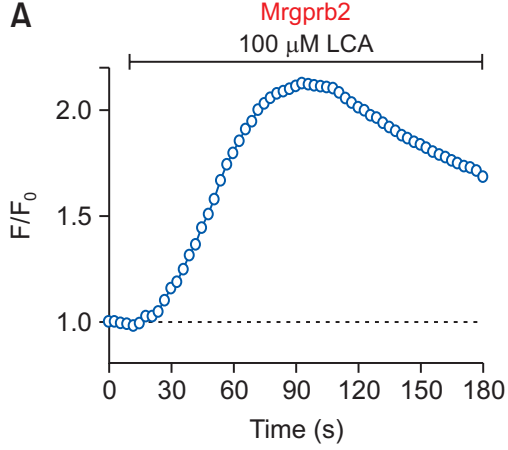

D

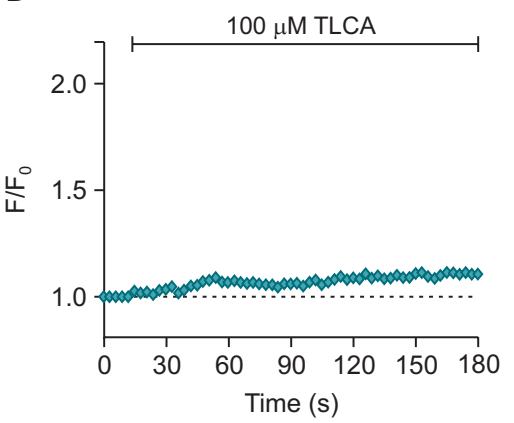

B

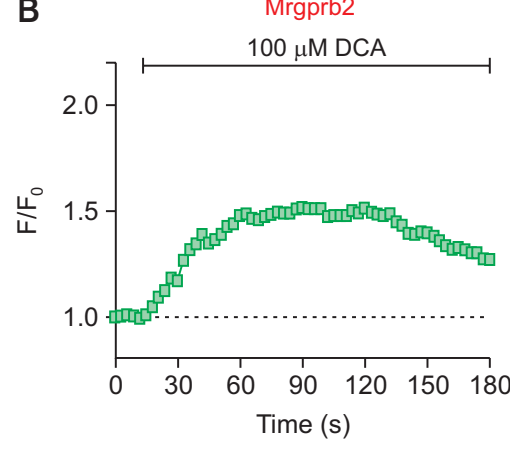

E

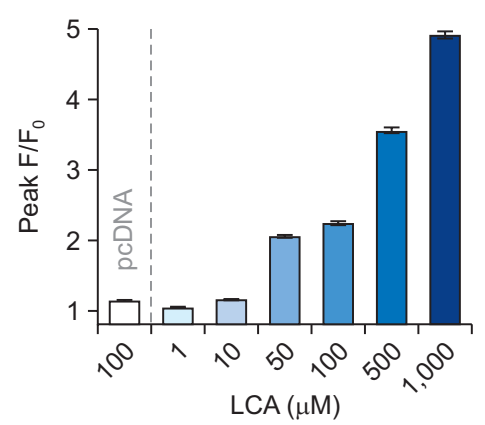

C

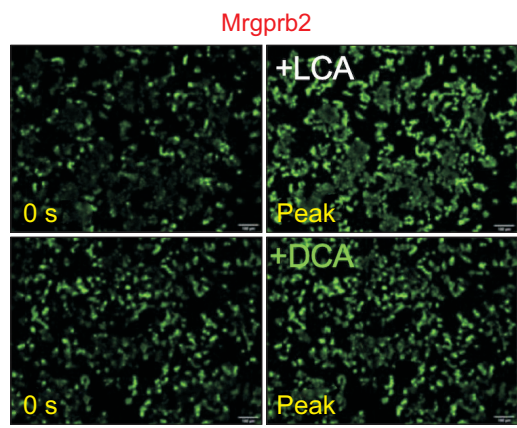

$\mathrm{F}$

Mrgprb2

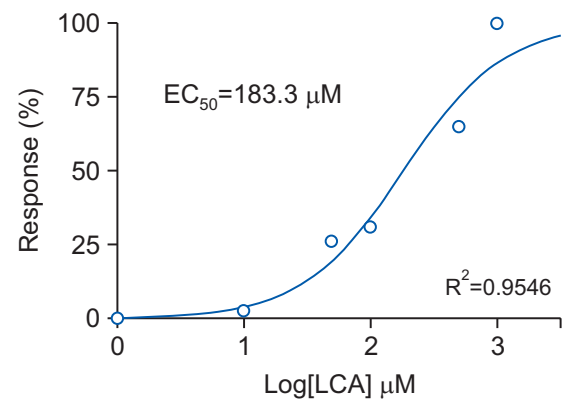

Fig. 3. Lithocholic acid (LCA) increases intracellular calcium levels through Mrgprb2. (A, B) HEK293T cells transiently expressing mouse Mrgprb2 (Mrgprb2-HEK293T) were treated with either LCA $(100 \mu \mathrm{M})$ and DCA $(100 \mu \mathrm{M})$. $(n=2,350$ cells and $n=3211$ cells, respectively) $(C)$ Representative fluorescent images of Mrgprb2-HEK293T at initial (0 s) and peak time points. Scale bars=100 $\mu \mathrm{m}$. (D) Taurolithocholic acid (TLCA; $100 \mu \mathrm{M}$ ) induced marginal responses in Mrgprb2-HEK293T. ( $n=3,164$ cells) (E) A summary of peak $F / F_{0}$ from the Mrgprb2-HEK293T after treatment of different concentration ranges of LCA. Cells without Mrgprb2 expression did not show an increase in intracellular calcium levels ( $p c D N A, n=1,684$ cells). ( $F)$ Concentration-effect curves with LCA on Mrgprb2-HEK293T $\left(R^{2}=0.9546\right)$. 
A
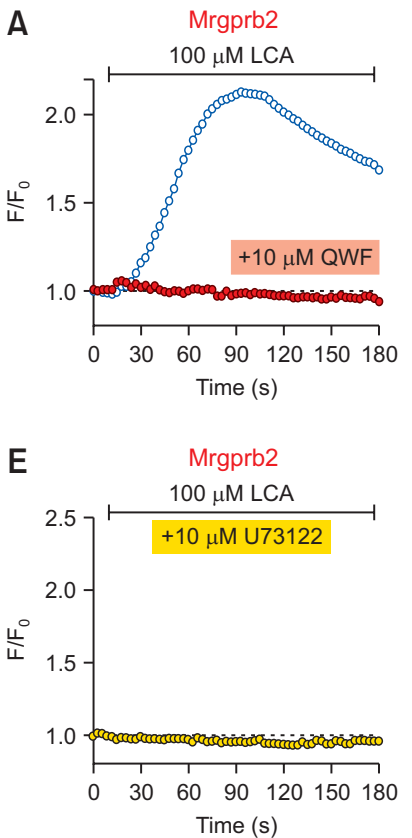
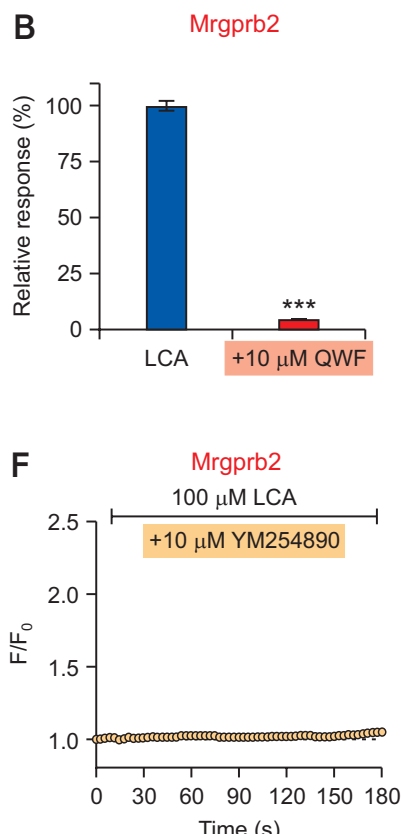
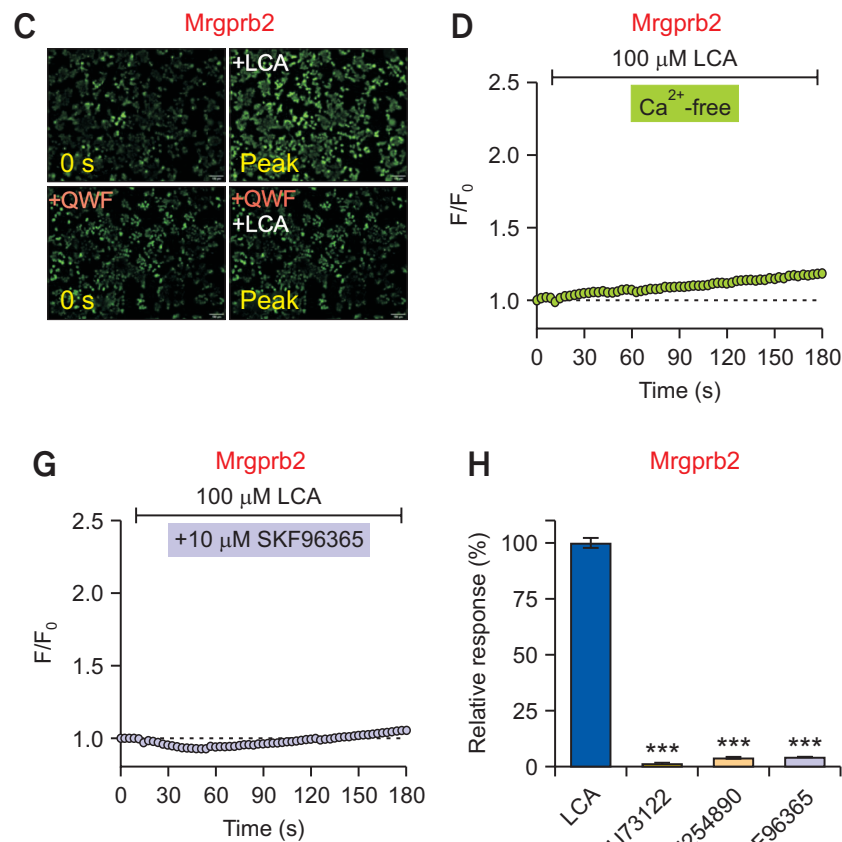

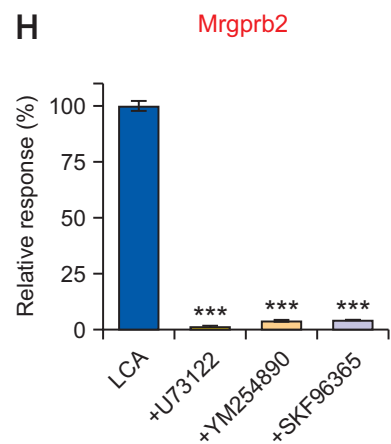

Fig. 4. The lithocholic acid (LCA)-induced response is specifically mediated by Mrgprb2. (A) LCA-induced increase in intracellular calcium ( $n=2,350$ cells) was suppressed by pretreatment with $10 \mu \mathrm{M}$ QWF (1,470 cells) in Mrgprb2-expressing HEK293T cells (Mrgprb2-HEK293T). (B) A comparison of relative responses between LCA vs. QWF pretreatment. ${ }^{* * *} p<0.001$. (C) Representative fluorescent images of Mrgprb2HEK293T at initial (0 s) and peak time points for QWF pretreatment before LCA application. Scale bars=100 $\mu \mathrm{m}$. (D) LCA did not evoke a noticeable increase in intracellular calcium levels in calcium-free media ( $n=3,393$ cells). (E-G) Responses from various inhibitors on LCA treatment in Mrgprb2-HEK293T. LCA-induced responses were significantly suppressed by SKF96365 (E; a non-specific TRPC blocker, $n=2,276$ cells), U73122 ( $F$; an inhibitor of PLC, $n=1,078$ cells), and YM254890 (G; a $G \alpha_{q}$ inhibitor, $n=1,314$ cells). (H) A summary of relative responses of pretreatment effects with various inhibitors in the Mrgprb2-HEK293T. ${ }^{* * *} p<0.001$.

induced response is indeed mediated by Mrgprb2. Similar to Mrgpra1, the same inhibitors used in Fig. 2 were applied again, and the response in Mrgprb2-HEK293T also showed an identical pattern. In detail, LCA did not induce responses in calcium-free media in Mrgprb2-HEK293T (Fig. 4D), and the activation of Mrgprb2 by LCA was completely abolished by pretreatment with U73122 (a PLC inhibitor, Fig. 4E, 4H), YM254890 (a G $\alpha_{q}$ inhibitor, Fig. 4F, 4H), and SKF9665 (a TRPC inhibitor, Fig. 4G, 4H). Thus, these data strongly suggest that LCA can specifically activate Mrgprb2 as well.

\section{LCA can activate mouse sensory neurons probably via Mrgpra 1}

Considering that LCA activates Mrgpra1, we further investigated whether LCA can activate mouse sensory neurons because Mrgpra1, but not Mrgprb2, is expressed in sensory neurons (Meixiong et al., 2019a, 2019b). Thus, we used primary cultures of mouse DRG neurons to investigate whether LCA can excite sensory neurons in a Mrgpra1-dependent manner using calcium imaging techniques.

As shown in Fig. 5A, 5B, and $5 \mathrm{C}, 100 \mu \mathrm{M}$ of LCA induced significantly increased responses in mouse DRG neurons. More importantly, the calcium increase by LCA was significantly inhibited by pretreatment with $10 \mu \mathrm{M}$ of QWF, indicating that LCA-induced intracellular calcium increase is probably due to the activation of Mrgpra1 (Fig. 5A-5C). To further verify the effect of LCA on DRG rigorously, LCA was applied twice: the first treatment was to verify if the cultured cells were functionally sensitive to LCA while the second treatment was to investigate if the responses could be inhibited by QWF pretreatment. Consequently, all cultured DRG neurons showed comparable responses after the first treatment with $100 \mu \mathrm{M}$ LCA (data not shown). After verifying the responses to the first LCA treatment, DRG neurons were pretreated with $10 \mu \mathrm{M}$ QWF, followed by a second treatment with $100 \mu \mathrm{M}$ LCA. As shown in Fig. 5D, QWF pretreatment significantly inhibited the response induced by the second LCA application, resulting in more than $50 \%$ inhibition compared to the control (Fig. 5E). Therefore, these data strongly imply that LCA can activate mouse DRG neurons in a Mrgpra1-dependent manner.

\section{LCA induces intracellular calcium increase in mouse PMCs}

Since Mrgprb2 is specifically found in mast cells (Meixiong et al., 2019a), we further investigated whether LCA can stimulate PMCs in an Mrgprb2-dependent manner. Calcium imaging experiments showed that LCA could evoke an increase in intracellular calcium levels in PMCs (Fig. 6A-6C). Moreover, the calcium increase induced by LCA was inhibited by pretreatment with QWF (Fig. 6A-6C), suggesting that LCA-induced responses in mast cells are mediated by Mrgprb2 activation.

To further verify the effect of LCA on mast cell degranulation, a $\beta$-hexosaminidase assay was performed. Although LCA induced mast cell degranulation in a dose-dependent manner (Fig. 6D), co-treatment with $10 \mu \mathrm{M}$ QWF and $100 \mu \mathrm{M}$ LCA did not induce any changes (data not shown). Therefore, although LCA can activate Mrgrpb2, mast cell degranulation in mouse 


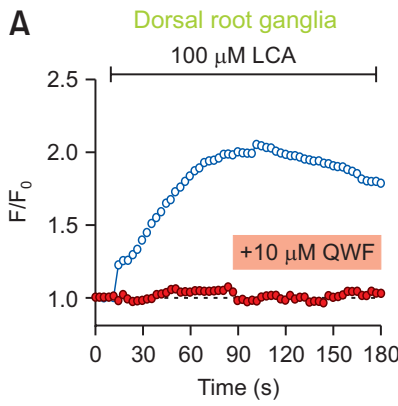

C

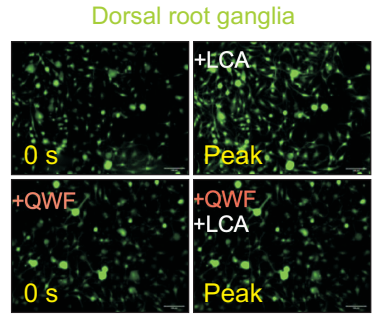

B Dorsal root ganglia

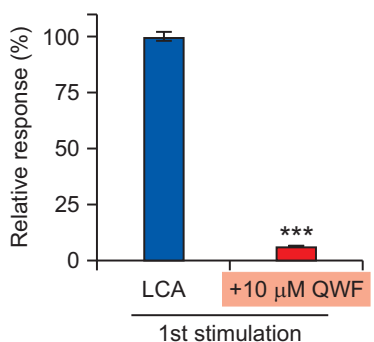

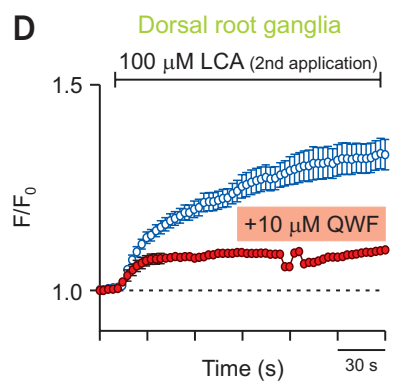

E

Dorsal root ganglia

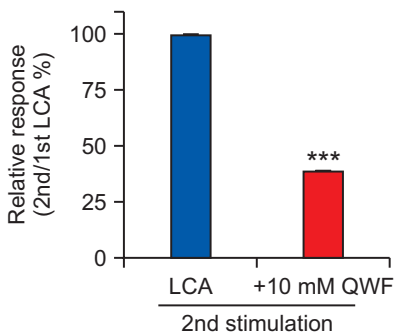

Fig. 5. Mrgpra1 mediates the effects of lithocholic acid (LCA) in sensory neurons. (A) Mouse dorsal root ganglia (DRG) neurons were treated with LCA $(100 \mu \mathrm{M})$. An LCA-induced increase in intracellular calcium $(n=2,558$ cells) was suppressed by pretreatment of $10 \mu \mathrm{M}$ QWF ( $n=701$ cells) in mouse DRG neurons. (B) A comparison of relative responses between LCA vs. QWF pretreatment. ${ }^{* * *} p<0.001$. (C) Representative fluorescent images of the mouse DRG neurons at initial $(0 \mathrm{~s})$ and peak time points for QWF pretreatment before LCA application. Scale bars $=100 \mu \mathrm{m}$. (D) DRG neurons responded to subsequent LCA exposure ( $\mathrm{n}=155$ cells). QWF $(10 \mu \mathrm{M})$ blocked LCA-induced (100 $\mu \mathrm{M})$ calcium increase in LCA-responding DRG neurons ( $n=169$ cells). $\mathrm{KCl}(100 \mathrm{mM})$ solution was also used to identify viable cells (data not shown). (E) QWF $(10 \mu \mathrm{M})$ significantly blocked second stimulation of LCA-induced $(100 \mu \mathrm{M})$ calcium flux in DRG neurons. ${ }^{* * *} p<0.001$.
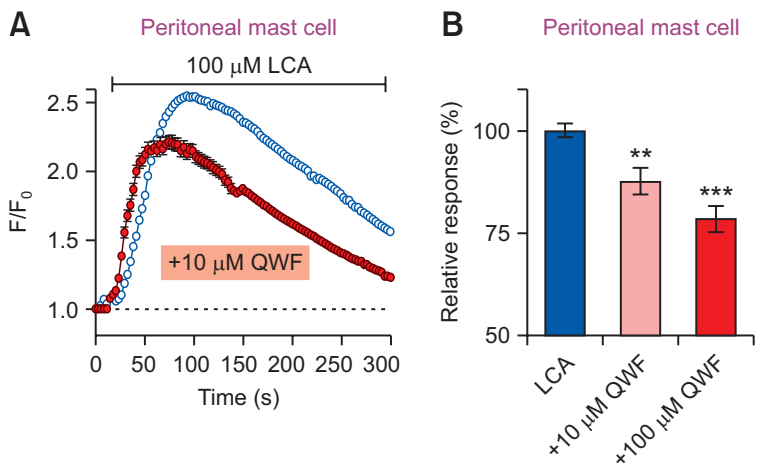

C Peritoneal mast cell

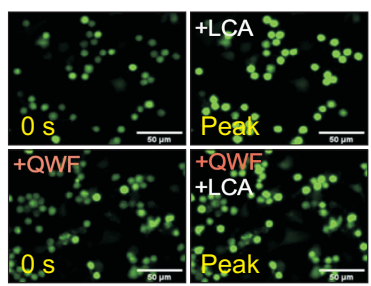

D

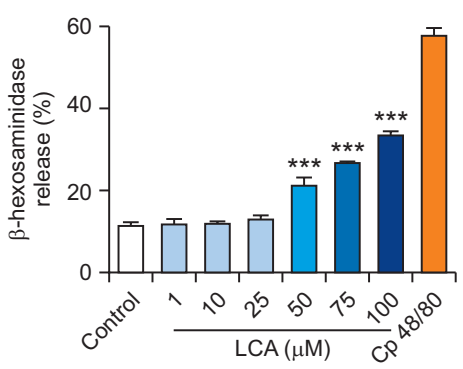

Fig. 6. Mrgprb2 mediates the effects of lithocholic acid (LCA) in peritoneal mast cells (PMCs). (A) Mouse PMCs were treated with LCA (100 $\mu \mathrm{M})$. An LCA-induced increase in intracellular calcium ( $\mathrm{n}=2038$ cells) was suppressed by pretreatment with $100 \mu \mathrm{M}$ QWF in mouse PMCs $(n=877$ cells). (B) A comparison of relative responses of cells treated with LCA alone (left), LCA pretreated with $10 \mu M$ QWF (middle), or 100 $\mu \mathrm{M}$ QWF (right). (C) Representative fluorescent images of the PMCs at initial $(0 \mathrm{~s})$ and peak time points for $100 \mu \mathrm{M}$ QWF pretreatment before LCA application. Scale bars $=50 \mu \mathrm{m}$. (D) Results from $\beta$-hexosaminidase assay on PMCs treated with various LCA concentrations. Cp $48 / 80$ indicates treatment of $100 \mu \mathrm{g} / \mathrm{mL}$ compound $48 / 80 .{ }^{* *} p<0.01$ and ${ }^{* * *} p<0.001$.

PMCs might occur via unknown LCA-mediated pathway(s) other than via Mrgprb2.

\section{Mrgpra1/b2 antagonist affects LCA-induced scratching behavior in mice}

Finally, the pruritogenic effect of LCA was tested by in vivo mouse scratching behavior test. As shown in Fig. 7A, acute intradermal injection of LCA into the cheek of mice did not significantly increase the total bouts of scratching (Fig. 7A), suggesting that LCA per se is not a potent pruritogen in mice. However, when the bouts of scratching were analyzed at 1-min intervals, a difference was noted at a specific time point that showed a difference (Fig. 7C). Thus, the data imply that LCA might be a weak pruritogen since the pruritogenic effect was only recorded at certain time points.

When $500 \mu \mathrm{M}$ of QWF, an antagonist of Mrgpra1 and Mrgprb2, was intradermally administered along with LCA, the total bouts of scratching did not change as well (Fig. 7A). Neverthe- 

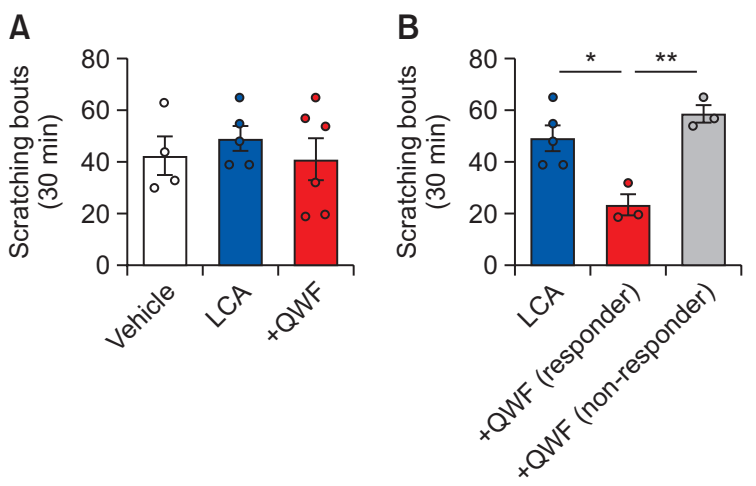

C

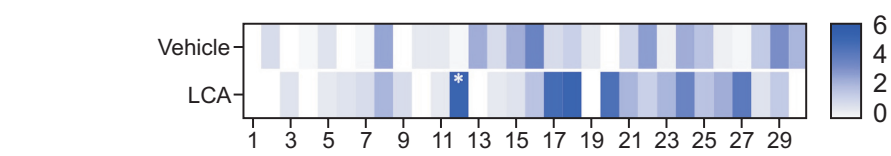

D

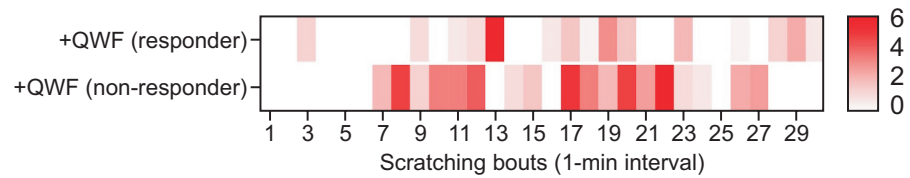

Fig. 7. Lithocholic acid (LCA) induces mild scratching behavior in both cheek models. (A) Intradermal injection of LCA into the cheek (50 $\mu \mathrm{g} / \mathrm{site}, \mathrm{n}=5$ ) did not induce significant changes in total bouts of scratching compared to vehicle $(\mathrm{n}=5)$. Additionally, co-administration of LCA and QWF $(500 \mu \mathrm{M}, \mathrm{n}=6)$ did not affect the total bouts of scratching. (B) QWF induced mixed responses in mice when co-treated with LCA, resulting in decreased scratching bouts in one group (responder) or no responses in the other group (non-responder). Heatmap data of scathing bouts per 1-min interval showing the time course of scratching behavior induced by intradermal injection of LCA alone (C) or cotreatment with QWF (D). Different scratching profiles among groups, which are not easily detected when the data are only presented as total bouts of scratching. ${ }^{*} p<0.05$.

less, two different groups of mice showed drastically different sensitivities to LCA. In other words, "responder" mice showed decreased scratching bouts after QWF treatment, whereas "non-responder" mice did not show any difference (Fig. 7B). Moreover, when the bouts of scratching were analyzed at 1-min intervals, these two groups showed dramatically different patterns of scratching bouts (Fig. 7D). Despite the discrepancy, some groups of mice responded to QWF co-treatment against LCA-induced scratching behavior. Thus, it was found that Mrgpra1/b2 antagonist QWF may affect LCA-induced scratching behavior in mice.

In conclusion, the present study is the first to show that LCA can activate both Mrgpra1 and Mrgprb2. We further verified that LCA can activate both sensory neurons and mast cells via Mrgpra1 and Mrgprb2, respectively. Although the pruritogenic role of LCA in non-cholestatic conditions could be minimal, the current findings clearly provide an insight into the similarities and differences of MRGPR families between humans and mice, paving a way to understand the complex roles of these pruriceptors.

\section{DISCUSSION}

Generally, LCA is regarded as a toxic compound that can induce liver damage. Oral administration of LCA into mice causes cholestatic liver injury via the JNK/STAT3 signaling pathway (Xu et al., 2020). Due to its detrimental effects, conventional LCA-related studies have focused on LCA-induced hepatotoxicity, which may account for the pathogenesis of liver injury and/or bile duct obstruction under cholestatic conditions (Fickert et al., 2006; Woolbright et al., 2014). Thus, various LCA studies have focused on the mechanisms and/ or pathways of LCA required for its elimination, excretion, and detoxification. The detoxification of bile acids, including LCA, is mostly achieved by CYP3A4, a major cytochrome P450 (Chen et al., 2014). Furthermore, the LCA-induced liver damage can be protected by activation of pregnane $X$ receptor (PXR) (Staudinger et al., 2001). Similarly, Nrf2 plays a crucial cytoprotective role against LCA-induced liver injury (Tan et al.,
2010). Overall, these reports indicate LCA as a hepatotoxic bile acid that must be readily eliminated.

The present study, however, started on the premise that LCA may play a role as a pruritogen, especially under cholestatic conditions. Indeed, bile acids are recognized as pruritogens in cholestasis. In detail, bile acids such as CA, CDCA, taurochenodeoxycholic acid (TCDCA), and LCA induced a marginally increased, albeit significant, itch sensation in humans (Yu et al., 2019). Most importantly, the best example of pruritogenic bile acid is DCA, since it induces significantly enhanced itch sensation in both mice and humans (Alemi et al., 2013; Yu et al., 2019). Moreover, the underlying molecular entities necessary for pruritus were also identified. Indeed, TGR5 (also known as $\mathrm{G}$ protein-coupled bile acid receptor 1 [GPBAR1]), a G-protein coupled receptor expressed in sensory neurons, mediates DCA-induced itch in mice (Alemi et al., 2013). The intradermal injection of TLCA and oleanolic acid induced itching in a TGR5-dependent manner since the responses were markedly reduced in TGR5 knockout mice (Alemi et al., 2013). However, it is unclear whether the activation of TGR5 directly induces pruritus at the sensory neuronal level, since human TGR5 was found to be expressed in satellite glial cells, contrary to the specific expression of mouse TGR5 in DRG neurons (Yu et al., 2019). Moreover, betulinic acid, which is a selective TGR5 agonist, failed to elicit an itch response in mouse cholestasis models induced by $\alpha$-naphthyl isothiocyanate (ANIT) (Cipriani et al., 2015), increasing the uncertainty of TGR5 as a pruriceptor expressed in sensory neurons.

Recently, several studies have determined that bile acids can also activate MRGPRX4, a member of the MRGPR responsible for cholestatic pruritus in humans (Meixiong et al., 2019c; Yu et al., 2019). Interestingly, DCA is the most potent bile acid that can activate MRGPRX4 (Meixiong et al., 2019c; Yu et al., 2019). Meanwhile, LCA can also weakly activate MRGPRX4, which may explain why LCA induces a weak, albeit evident, itch sensation in humans (Yu et al., 2019). However, unlike DCA, no details of molecular underpinnings, through which LCA contributes to itch sensation in mice, were found. Although Mrgpra1 has been considered as a functional counterpart of human MRGPRX4, the present study is the first 
to confirm that Mrgpra1 indeed reacts with LCA. Surprisingly, LCA can also activate Mrgrpb2, which is acknowledged as a functional counterpart of human MRGPRX2, but not of MRGPRX4.

Although LCA can activate both Mrgpra1 and Mrgprb2, it seems odd that a certain agonist can activate two different receptors. However, these dual agonists, especially for both Mrgpra1 and Mrgprb2, have already been reported. For instance, substance $P$, an inflammatory neuropeptide and potent endogenous pruritogen, can activate both Mrgpra1 (Azimi et al., 2016, 2017) and Mrgprb2 (McNeil et al., 2015; Azimi et al., 2016; Green et al., 2019). Similarly, compound $48 / 80$ is also known to activate both Mrgpra1 and Mrgprb2 (Azimi et al., 2016), and the dual activation effect of compound $48 / 80$ was confirmed in our study (Supplementary Fig. 2). Although the three-dimensional protein structures of MRGPRs have not yet been revealed, it is speculated that Mrgpra1 and Mrgprb2 may have similar binding pockets for agonists since Mrgpra1 and Mrgprb2 share similar amino acid sequences (Supplementary Fig. 1A). Hopefully, further structural studies of MRGPR will reveal the underlying mechanisms of how these receptors can bind to the same agonist.

While Mrgpra1 and Mrgprb2 share various similarities, the expression profiles of these receptors are entirely different. For example, Mrgpra1, but not Mrgprb2, is expressed in bone marrow-derived dendritic cells (BMDCs) (Perner et al., 2020). Additionally, Mrgprb2 is specifically expressed in mast cells (Meixiong et al., 2019a), whereas Mrgpra1 is found in a subset of sensory neurons that innervate the skin and ramify into lamina I and II of the spinal cord (Meixiong et al., 2019b). In general, it appears that Mrgpra1 is mostly found in sensory neurons, while Mrgprb2 is specifically expressed in mast cells. This is further supported by the results shown in Fig. 5 and 6 that LCA activates mouse sensory neurons (DRGs) and mast cells (PMCs), probably via Mrgpra1- and Mrgprb2-dependent pathways, respectively. While DRG activation can directly trigger itch sensation, mast cell activation leads to its degranulation, which in turn induces the release of endogenous pruritogens, such as histamine and tryptase, to cause pruritus. Overall, the present study supports the idea that LCA can contribute to itch through sensory neuron activation and/or mast cell degranulation by Mrgpra1 and Mrgprb2 activation, respectively.

Unfortunately, no difference in total bouts of scratching behaviors was found when LCA was intradermally administered into the cheek (Fig. 7). This result is in line with the fact that LCA-induced scratching behaviors in mice have not been reported to date. One probable reason could be that LCA per $s e$ is not a sufficiently potent pruritogen to evoke changes in scratching behaviors, at least in normal healthy ICR mice. However, the 1-min interval analysis in our study indicates LCA as a pruritogen (Fig. 7C). It is speculated that the putative pruritogenic role of LCA might be more visible under diseased conditions, such as cholestasis. Another plausible reason for the lack of changes in LCA-induced scratching behavior could be that mice do not show consistent responses to LCA. As shown in Fig. 7B and 7D, varying groups of ICR mice had different sensitivities to LCA, complicating the interpretation of the LCA effect. Although there is no proof, this is consistent with a similar report that not all patients with cholestasis develop pruritus (Sanjel and Shim, 2020). Further in-depth experiments are warranted to clarify the lack of strong scratching behavior changes in ICR mice.
Additionally, a difference in bile acid composition between humans and mice exists. Specifically, the major primary bile acids are CA and CDCA in humans, while they are CA and muricholic acid (MCA) in mice (Thakare et al., 2018). Importantly, this is further supported by the fact that LCA is relatively abundant in humans (Thakare et al., 2018) because LCA is a secondary bile acid derived from CDCA. Indeed, LCA is found to be less than $1 \%$ in normal mice (Thakare et al., 2018). Thus, it is speculated that the role of endogenous LCA in healthy mice is minimal. This may also explain why LCA failed to evoke scratching behavior in mice. In contrast, LCA induced (but not strong) itch sensation in humans.

The current findings warrant further in-depth investigation. For instance, a cholestasis animal model would be required to unravel the roles of Mrgpra1 and Mrgprb2 in mice. Moreover, studies in knockout mice, such as Mrgpra 1/ or Mrgprb2 ${ }^{-/}$, will help clarify uncertainties related to LCA-induced responses. We are currently planning to investigate these uncertainties in the future, which are beyond the scope of the present study. To the best of our knowledge, this is the first report to reveal that LCA activates Mrgpra1 and Mrgprb2, both of which contribute to itch sensation in mice. Moreover, the present study may provide an insight into MRGPR families, especially between humans and mice, paving the way to understand the complex roles of MRGPR members as a pruriceptor.

\section{ACKNOWLEDGMENTS}

This work was supported by the National Research Foundation of Korea (NRF) grant funded by the Korea government (MSIT) (No. 2021R1A2C1005865).

\section{REFERENCES}

Alemi, F., Kwon, E., Poole, D. P., Lieu, T., Lyo, V., Cattaruzza, F., Cevikbas, F., Steinhoff, M., Nassini, R., Materazzi, S., Guerrero-Alba, R., Valdez-Morales, E., Cottrell, G. S., Schoonjans, K., Geppetti, P., Vanner, S. J., Bunnett, N. W. and Corvera, C. U. (2013) The TGR5 receptor mediates bile acid-induced itch and analgesia. J. Clin. Invest. 123, 1513-1530.

Azimi, E., Reddy, V. B., Pereira, P. J. S., Talbot, S., Woolf, C. J. and Lerner, E. A. (2017) Substance P activates Mas-related G proteincoupled receptors to induce itch. J. Allergy Clin. Immunol. 140, 447-453.e3.

Azimi, E., Reddy, V. B., Shade, K. C., Anthony, R. M., Talbot, S., Pereira, P. J. S. and Lerner, E. A. (2016) Dual action of neurokinin-1 antagonists on Mas-related GPCRs. JCI Insight 1, e89362.

Carey, J. B., Jr., Wilson, I. D., Zaki, F. G. and Hanson, R. F. (1966) The metabolism of bile acids with special reference to liver injury. Medicine (Baltimore) 45, 461-470.

Chen, J., Zhao, K. N. and Chen, C. (2014) The role of CYP3A4 in the biotransformation of bile acids and therapeutic implication for cholestasis. Ann. Transl. Med. 2, 7.

Cipriani, S., Renga, B., D'Amore, C., Simonetti, M., De Tursi, A. A., Carino, A., Monti, M. C., Sepe, V., Zampella, A. and Fiorucci, S. (2015) Impaired itching perception in murine models of cholestasis is supported by dysregulation of GPBAR1 signaling. PLOS ONE 10, e0129866.

Dong, X., Han, S., Zylka, M. J., Simon, M. I. and Anderson, D. J. (2001) A diverse family of GPCRs expressed in specific subsets of nociceptive sensory neurons. Cell 106, 619-632.

Fickert, P., Fuchsbichler, A., Marschall, H. U., Wagner, M., Zollner, G., Krause, R., Zatloukal, K., Jaeschke, H., Denk, H. and Trauner, M. (2006) Lithocholic acid feeding induces segmental bile duct ob- 
struction and destructive cholangitis in mice. Am. J. Pathol. 168, 410-422.

Fisher, M. M., Magnusson, R. and Miyai, K. (1971) Bile acid metabolism in mammals. I. Bile acid-induced intrahepatic cholestasis. Lab. Invest. 25, 88-91.

Green, D. P., Limjunyawong, N., Gour, N., Pundir, P. and Dong, X. (2019) A mast-cell-specific receptor mediates neurogenic inflammation and pain. Neuron 101, 412-420.e3.

Han, S. K., Dong, X., Hwang, J. I., Zylka, M. J., Anderson, D. J. and Simon, M. I. (2002) Orphan G protein-coupled receptors MrgA1 and MrgC11 are distinctively activated by RF-amide-related peptides through the Galpha q/11 pathway. Proc. Natl. Acad. Sci. U.S.A. 99, $14740-14745$.

Islam, M. N., Lee, K. W., Yim, H. S., Lee, S. H., Jung, H. C., Lee, J. H. and Jeong, J. Y. (2017) Optimizing T4 DNA polymerase conditions enhances the efficiency of one-step sequence- and ligationindependent cloning. Biotechniques 63, 125-130.

Jeong, J. Y., Yim, H. S., Ryu, J. Y., Lee, H. S., Lee, J. H., Seen, D. S. and Kang, S. G. (2012) One-step sequence- and ligation-independent cloning as a rapid and versatile cloning method for functional genomics studies. Appl. Environ. Microbiol. 78, 5440-5443.

Lay, M. and Dong, X. (2020) Neural mechanisms of itch. Annu. Rev. Neurosci. 43, 187-205.

McNeil, B. D., Pundir, P., Meeker, S., Han, L., Undem, B. J., Kulka, M. and Dong, X. (2015) Identification of a mast-cell-specific receptor crucial for pseudo-allergic drug reactions. Nature 519, 237-241.

Meixiong, J., Anderson, M., Limjunyawong, N., Sabbagh, M. F., Hu, E., Mack, M. R., Oetjen, L. K., Wang, F., Kim, B. S. and Dong, X. (2019a) Activation of mast-cell-expressed mas-related G-proteincoupled receptors drives non-histaminergic itch. Immunity $\mathbf{5 0}$ 1163-1171.e5.

Meixiong, J., Vasavda, C., Green, D., Zheng, Q., Qi, L., Kwatra, S. G., Hamilton, J. P., Snyder, S. H. and Dong, X. (2019b) Identification of a bilirubin receptor that may mediate a component of cholestatic itch. Elife 8, e44116.

Meixiong, J., Vasavda, C., Snyder, S. H. and Dong, X. (2019c) MRGPRX4 is a $\mathrm{G}$ protein-coupled receptor activated by bile acids that may contribute to cholestatic pruritus. Proc. Natl. Acad. Sci. U.S.A. 116, 10525-10530.

Perner, C., Flayer, C. H., Zhu, X., Aderhold, P. A., Dewan, Z. N. A., Voisin, T., Camire, R. B., Chow, O. A., Chiu, I. M. and Sokol, C. L. (2020) Substance $P$ release by sensory neurons triggers dendritic cell migration and initiates the type- 2 immune response to aller- gens. Immunity 53, 1063-1077.e7.

Pradhananga, S. and Shim, W. S. (2015) Caffeic acid exhibits antipruritic effects by inhibition of multiple itch transmission pathways in mice. Eur. J. Pharmacol. 762, 313-321.

Ridlon, J. M., Harris, S. C., Bhowmik, S., Kang, D. J. and Hylemon, P. B. (2016) Consequences of bile salt biotransformations by intestinal bacteria. Gut Microbes 7, 22-39.

Sanjel, B. and Shim, W. S. (2020) Recent advances in understanding the molecular mechanisms of cholestatic pruritus: a review. Biochim. Biophys. Acta Mol. Basis Dis. 1866, 165958.

Staudinger, J. L., Goodwin, B., Jones, S. A., Hawkins-Brown, D., MacKenzie, K. I., LaTour, A., Liu, Y., Klaassen, C. D., Brown, K. K., Reinhard, J., Willson, T. M., Koller, B. H. and Kliewer, S. A. (2001) The nuclear receptor PXR is a lithocholic acid sensor that protects against liver toxicity. Proc. Natl. Acad. Sci. U.S.A. 98, 3369-3374.

Subramanian, H., Gupta, K. and Ali, H. (2016) Roles of Mas-related G protein-coupled receptor $\mathrm{X} 2$ on mast cell-mediated host defense, pseudoallergic drug reactions, and chronic inflammatory diseases. J. Allergy Clin. Immunol. 138, 700-710.

Tan, K. P., Wood, G. A., Yang, M. and Ito, S. (2010) Participation of nuclear factor (erythroid 2-related), factor 2 in ameliorating lithocholic acid-induced cholestatic liver injury in mice. Br. J. Pharmacol. 161, 1111-1121.

Thakare, R., Alamoudi, J. A., Gautam, N., Rodrigues, A. D. and Alnouti, Y. (2018) Species differences in bile acids I. Plasma and urine bile acid composition. J. Appl. Toxicol. 38, 1323-1335.

Tsvilovskyy, V., Solis-Lopez, A., Ohlenschlager, K. and Freichel, M. (2018) Isolation of peritoneum-derived mast cells and their functional characterization with $\mathrm{Ca} 2+-$ imaging and degranulation assays. J. Vis. Exp. (137), 57222.

Woolbright, B. L., Li, F., Xie, Y., Farhood, A., Fickert, P., Trauner, M. and Jaeschke, H. (2014) Lithocholic acid feeding results in direct hepato-toxicity independent of neutrophil function in mice. Toxicol. Lett. 228, 56-66.

Xu, G., Dai, M., Zheng, X., Lin, H., Liu, A. and Yang, J. (2020) Cholestatic models induced by lithocholic acid and alphanaphthylisothiocyanate: different etiological mechanisms for liver injury but shared JNK/STAT3 signaling. Mol. Med. Rep. 22, 1583-1593.

Yu, H., Zhao, T., Liu, S., Wu, Q., Johnson, O., Wu, Z., Zhuang, Z., Shi, Y., Peng, L., He, R., Yang, Y., Sun, J., Wang, X., Xu, H., Zeng, Z., Zou, P., Lei, X., Luo, W. and Li, Y. (2019) MRGPRX4 is a bile acid receptor for human cholestatic itch. Elife 8, e48431. 